\title{
Appropriateness of the post-operative rehabilitation of low energy hip fractures in elderly in comparison with the AAOS appropriate use criteria at a level one trauma center
}

\author{
Mohammed Adam ${ }^{1} \cdot$ Eslam Alkaramany $^{1} \cdot$ Abduljabbar Alhamoud $^{1} \cdot$ Jawad Derbas $^{1}$ - Abdullah Murshid ${ }^{1}$. \\ Ghalib Ahmed Alhaneedi ${ }^{1}$ (I)
}

Received: 4 December 2020 / Accepted: 10 March 2021 / Published online: 30 March 2021

(c) The Author(s) 2021

\begin{abstract}
Purpose This study aimed to assess the appropriateness of the post-operative rehabilitation of low energy hip fractures in the elderly by comparing between the rehabilitations actually provided at level one trauma center and the AAOS Appropriate Use Criteria (AUC) recommendations.

Methods A retrospective review of the medical charts of all patients who underwent surgery for hip fractures followed by post-operative rehabilitation between October 2016 and May 2018. The age, gender, fracture types, four AUC variables including; the surgical approach, pre-operative mobility/functional status, cognitive impairment, and post-operative delirium, and types of post-operative rehabilitation received were collected.

The four patient variables were entered into the AUC application to generate the recommended rehabilitation procedures. Afterward, the rate of appropriateness of the treatments and the agreement between the rehabilitations actually provided and the AUC recommendation were measured.

Results Over the study period, a consecutive series of 101 patients were included. The mean age was 75 years. Most of the patients were males $(51.5 \%)$. Seventeen scenarios were observed in our patients. The most common scenario were patients with low functional/physical demands (48\%), intact cognitive function (91\%), non-arthroplasty approach (76\%), and no post-operative delirium(97\%).

The overall appropriateness rate of the provided rehabilitation treatments for our patients in comparison with AUC recommendation was appropriate in $356(48.7 \%)(P=.001)$, maybe appropriate in $19(3 \%)(P<.001)$, rarely appropriate in 61 $(8.3 \%)(P=.59)$, and $40 \%$ of rehabilitation procedures were not provided $(P<.001)$.

The actual treatment was appropriate and in agreement with the AUC recommendations in (100\%) of three procedures (Deep venous thrombosis prophylaxis, pain management, and Inpatient Rehabilitation Facility or Skilled Nursing Facility), in (72.2\%) of osteoporosis assessment/management, in (63.8\%) of outpatient occupational/physical therapy, in (10.2\%) of delirium prevention, in $(33.3 \%)$ of delirium management and in $(25 \%)$ of home care therapy.

Conclusions This study demonstrated that there is a remarkable variation in the appropriateness of the various post-operative rehabilitation procedures for elderly hip fracture. Additionally, the AUC application was easy to use and simple for identifying post-operative rehabilitation protocols for elderly hip fractures, hence, we recommend to use it in the trauma clinical practice. Level of evidence: IV
\end{abstract}

Keywords American Academy of Orthopedic Surgeons (AAOS) - Appropriate Use Criteria $\cdot$ Elderly $\cdot$ Hip fracture $\cdot$ Postoperative rehabilitation - Trauma center

Ghalib Ahmed Alhaneedi

ghaliboudah@gmail.com; gahmed@hamad.qa

1 Orthopedics Department, Hamad General Hospital, Hamad Medical Corporation, PO Box 3050, Doha, Qatar

\section{Introduction}

Hip fracture is one of the most common fractures in the Elderly. It is usually caused by low-energy trauma. It is often associated with osteoporosis and other medical comorbidities that may increase the prevalence of falls [1-3]. 
Most fractures occur in women older than 65 years, with an estimated worldwide incidence of approximately 1.7 million per year [2, 3].

Elderly patients with hip fractures are at risk for decreased level of mobility, inability to return to prior living circumstances, impaired quality of life, and increased rate of mortality [2-9].

Hip fractures are almost always treated surgically with either internal fixation or arthroplasty depending on the fracture type, age of the patient, fracture site, and pre-injury functional status $[10,11]$. The post-operative rehabilitation of hip fractures in the elderly depends on several factors, including fracture type and surgery, the patient-related factors such as pre-operative mobility/functional status, cognitive impairment and post-operative delirium [12]. Postoperative physiotherapy and occupational therapy mainly focus on the function, the mobility of the patient, daily life activities, and independent living [12-14].

To improve the quality of the management of hip fractures in the elderly, the American Academy of Orthopedic Surgeons (AAOS) published clinical practice guidelines in 2014 based on the best available evidence [2,3].

Subsequently, the AAOS published the Appropriate Use Criteria (AUC) in 2015 for the Post-operative Rehabilitation of Low Energy Hip Fractures in the Elderly (PORHFE) based on the relevant evidence and experts from different fields of musculoskeletal care. The AAOS made the free web-based AUC application widely available to help orthopedic surgeons to select the most appropriate post-operative rehabilitation protocols $[15,16]$.

Four clinical variables for a specific patient clinical scenario are entered into the AUC application: a surgical approach for arthroplasty (or non-arthroplasty); pre-operative mobility/functional status; cognitive impairment; and postoperative delirium. The application then provides a listing of upto 10 different rehabilitation procedure recommendations. Each procedure is rated into one of three categories: appropriate, may be appropriate, and rarely appropriate $[15,17]$.

This study aimed to assess the appropriateness of the post-operative rehabilitation of low energy hip fractures in the elderly by comparing between the rehabilitations actually provided at a level one trauma center and the AAOS Appropriate Use Criteria (AUC) recommendations.to determine the rate of appropriateness and agreement with AUC recommendations.

\section{Methods and materials}

\section{Study design and setting}

The Institutional Medical Research Center approved this study with a protocol number (MRC-01-18-072), and informed consent was exempted. Retrospectively, all elderly hip fractures ( $\geq 60$ years) who underwent surgical treatments (fixation or arthroplasty) between October 2016 and May 2018 were identified from the operating theater registry. Our institution is a level 1 trauma center accredited by Joint Commission International (JCI) and Accreditation Council of Graduate Medical Education-International (ACGME-I). Our orthopedic unit includes 14 consultant orthopedic surgeons who manage orthopedic trauma, including hip fractures in elderly patients.

\section{Eligibility criteria}

The eligibility criteria were according to the AUC criteria for post-operative rehabilitation of hip fracture in elderly.

The inclusion criteria were patients who underwent surgery (fixation or arthroplasty) for low-energy isolated elderly hip fractures ( $\geq 60$ years).

Patients with previous surgical intervention, revision surgery, bilateral hip fractures, pathological fractures, and open fractures, poly trauma, and medical contraindications to post-operative rehabilitation therapies were excluded because those patients need special care and multidisciplinary approach which differs from the standard post-operative rehabilitation procedure.

\section{Data collection}

The data for patient age, gender, the surgical approach for arthroplasty or non-arthroplasty, pre-operative mobility/ functional status, cognitive impairment, post-operative delirium, and post-operative rehabilitation treatment procedures were collected from the patients' medical charts and radiographs.

The AUC for post-operative rehabilitation of low energy hip fractures in the elderly requires four patients' variables to generate appropriateness ratings for ten post-operative rehabilitation procedures. These variables include 1 . Surgical approach for arthroplasty, which was retrieved from surgeon operative note and could be either Posterior, Anterior/Anterolateral, or Non-arthroplasty procedure. 2. Preoperative Mobility/function status graded in four levels from high functioning to non-ambulatory. It was retrieved from admitting physician and physiotherapist notes. 3. Cognitive impairment was retrieved from an emergency physician triage or attending physician assessment notes. It was assessed using Mini-mental state examination (MMSE) and graded into three levels; intact, mild, and moderate or severe. 4. Post-operative delirium was collected from the post-operative attending physician or anesthetist assessment notes.

Thus, the four parameters according to the criteria of the AUC were retrieved by two authors for 101 consecutive patients included in this study and entered into the AUC 
application to generate the post-operative rehabilitation protocols.

To assess the appropriateness of post-operative rehabilitation, first, the four parameters of each patient were input into the AUC to generate the appropriateness rating of the ten provided rehabilitation procedures for each patient.

Each of the ten rehabilitation procedures is rated as appropriate, may be appropriate, or rarely appropriate according to the AUC application (Fig. 1 and Table 1).

Afterward, the appropriateness rate and agreement with the AUC recommendations were then compared between the treatments actually provided and the AUC recommendations.

The AAOS panels made amendments to remove the below treatment options from specific patient scenarios due to clinical irrelevance:

Delirium management was removed in scenarios with no post-operative delirium.

Delirium prevention was removed in patients with postoperative delirium.
Outpatient Rehabilitation was removed in the patient scenarios with the inability to leave the house (low function patient).

Outpatient Rehabilitation was removed in scenarios with non-ambulatory/bed dependent.

Interdisciplinary management at Inpatient Rehabilitation or Skilled Nursing Facility (IRF or SNF) was provided only if the patient unable to return home.

Home care therapy was provided if able to return home but unable to transport to a local rehab facility.

Thus, according to the above-mentioned amendments; two hundred seventy-eight rehabilitation procedures for 101 patients were removed. Hence, 732 rehabilitation procedures should be provided for our patients.

\section{Statistical analysis}

Descriptive statistics such as means, ranges, \pm standard deviation, and frequency were used to describe the continuous

\begin{tabular}{l} 
Indication Profile \\
Surgical Approach for Arthroplasty \\
Posterior \\
Anterior/Anterolateral \\
Non-arthroplasty Procedure \\
Pre-operative Mobility/Functional Status \\
High functioning/high physical demand patient \\
Not able to shop without assistance but able to leave house with or without \\
Notsistance- Moderate Function/ Normal to Moderate Demand Patient \\
Non-ambulatory/led bound/palliative - Very Low Function/Very Low \\
Physical Demand Patient \\
Cognitive Impairment \\
Intact (26-30 MmSE) \\
Mild cognitive dysfunction (20-25 MMSE) \\
Moderate or Severe cognitive dysfunction (less than 20 MMSE) \\
\hline Postoperative Delirium \\
No Postoperative Delirium \\
Postoperative Delirium
\end{tabular}

\section{Procedure Recommendations}

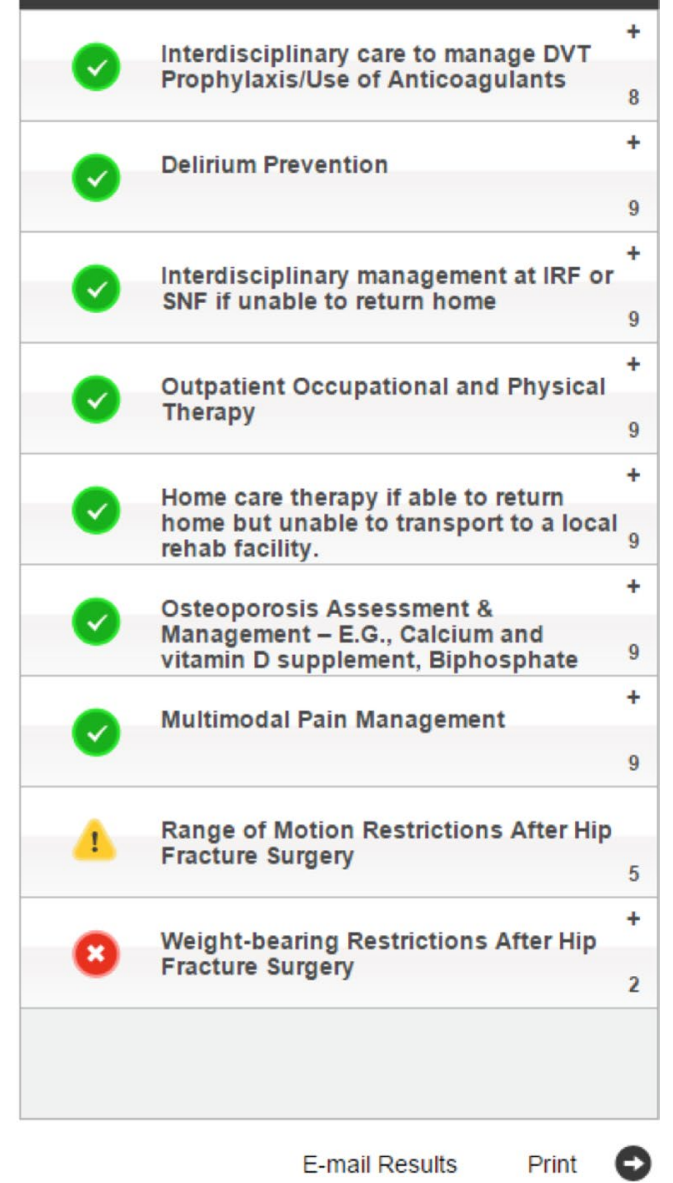

Fig. 1 Web-based AUC application screenshot [15] 
Table 1 Interpreting the final ratings of appropriate use criteria [15]

\begin{tabular}{lc}
\hline Level of appropriateness & Description \\
\hline $\begin{array}{l}\text { Appropriate } \\
\text { Maybe appropriate }\end{array}$ & $\begin{array}{l}\text { Median panel rating between } 7 \text { and } 9 \text { and no disagreement } \\
\text { Median panel rating between } 4 \text { and 6, or } \\
\text { Median panel rating 1-9 with disagreement } \\
\text { Median panel rating between } 1 \text { and } 3 \text { and no disagreement }\end{array}$ \\
$\begin{array}{l}\text { Rarely appropriate } \\
\text { Appropriate treatment is generally acceptable, is a reasonable approach for the indication, and is likely to improve the patients' health out- }\end{array}$ \\
$\begin{array}{l}\text { May be Appropriate treatment may be acceptable and may be a reasonable approach for the indication, but with uncertainty implying that more } \\
\text { research and/or patient information is needed to further classify the indication }\end{array}$ \\
$\begin{array}{l}\text { Rarely an appropriate option for management of patients in this population due to the lack of a clear benefit/risk advantage; rarely an effec- } \\
\text { tive option for individual care plans; exceptions should have documentation of the clinical reasons for proceeding with this care option (i.e., } \\
\text { procedure is not generally acceptable and is not generally reasonable for the indication) }\end{array}$ \\
\hline
\end{tabular}

variables such as the patient characteristics and patient scenarios. Whereas discrete variables were described as frequency (percentage of total) such as appropriateness rating for each rehabilitation procedure and the agreement of the treatments implemented at our institution with the AUC recommendations. Continuous and discrete variables were analyzed via $T$ test and Chi-squared test, respectively. A $p$ value $<0.05$ was considered statistically significant. All data were analyzed using statistical software (IBM SPSS version 22; SPSS Inc., Chicago, IL).

No sample size calculations were performed before conducting this study because all patients who met the inclusion criteria were included. A post hoc power analysis revealed a power of greater than $80 \%$, which indicated that the sample size was adequate for analysis. The calculation was based on using post-hoc calculator with a one sample proportion test and a 5\% level of significant to evaluate the power of existing study to detect a difference by comparing our appropriateness rate $(48.7 \%$ ) of 101 cases versus the AAOS AUC appropriateness rate of $75 \%$ that was published in previous literature.

\section{Results}

\section{Participants}

A total of 210 cases were identified in our theater registry with a diagnosis of hip fractures. One hundred and nine cases were excluded; the reasons for exclusion were $<60$ years in 80 patients, medical contraindication to post-operative rehabilitation in 13 cases, revision surgery in five patients, three cases were polytrauma, pathological fractures in seven patients and one patient with bilateral hip fractures. Thus, 101 patients with elderly hip fractures met the inclusion criteria and eligible for analysis (Fig. 2).

\section{Descriptive data}

The mean age was 75.6 years (range 60-96 y), and $51.5 \%$ were males. Forty-one case $(40.6 \%)$ sustained stable intertrochanteric, $23(22.8 \%)$ unstable intertrochanteric fractures, 7 (6.9\%) subtrochanteric, 26 (25.8\%) displaced neck of femur, 4 (3.9\%) nondisplaced neck of femur fractures.

Seventeen out of 72 AUC scenarios were observed in our study. With predominance of low function/low physical demands (48\%), intact cognitive function (91\%), nonarthroplasty approach $(76 \%)$ (stable intertrochanteric fracture $(40.5 \%)$ fixed by DHS $(44.5 \%)$ ) with no post-operative delirium (97\%). (Table 2).

\section{Outcome data}

In comparison with AUC recommendation, four hundred thirty-six $(60 \%)$ rehabilitation procedures were provided, and 296 (40\%) of rehabilitation procedures were not provided for our patients $(P<0.001)$.

The overall appropriateness rate of the provided rehabilitation treatments for our patients in comparison with AUC recommendation was appropriate in $356(48.7 \%)$ $(P=0.001)$, maybe appropriate in $19(3 \%)(P<0.001)$, and rarely appropriate in $61(8.3 \%)(P=0.59)$ of rehabilitation procedures.

The actual treatment was appropriate and in agreement with the AUC recommendations in (100\%) of three procedures (Deep venous thrombosis (DVT) prophylaxis, pain management, and Inpatient Rehabilitation Facility (IRF) or Skilled Nursing Facility (SNF)), in (72.2\%) of osteoporosis and assessment management $(P<0.01)$, in $(63.8 \%)$ of outpatient occupational and physical therapy $(P=0.01)$, in $(25 \%)$ of home care therapy $(P<0.001)$, in $(10.2 \%)$ of delirium prevention $(P<0.001)$, and in $(33.3 \%)$ of delirium management $(P=0.25)$. Table 3 and Fig. 3 summarize the rate of appropriateness and the agreement with AUC recommendations. 


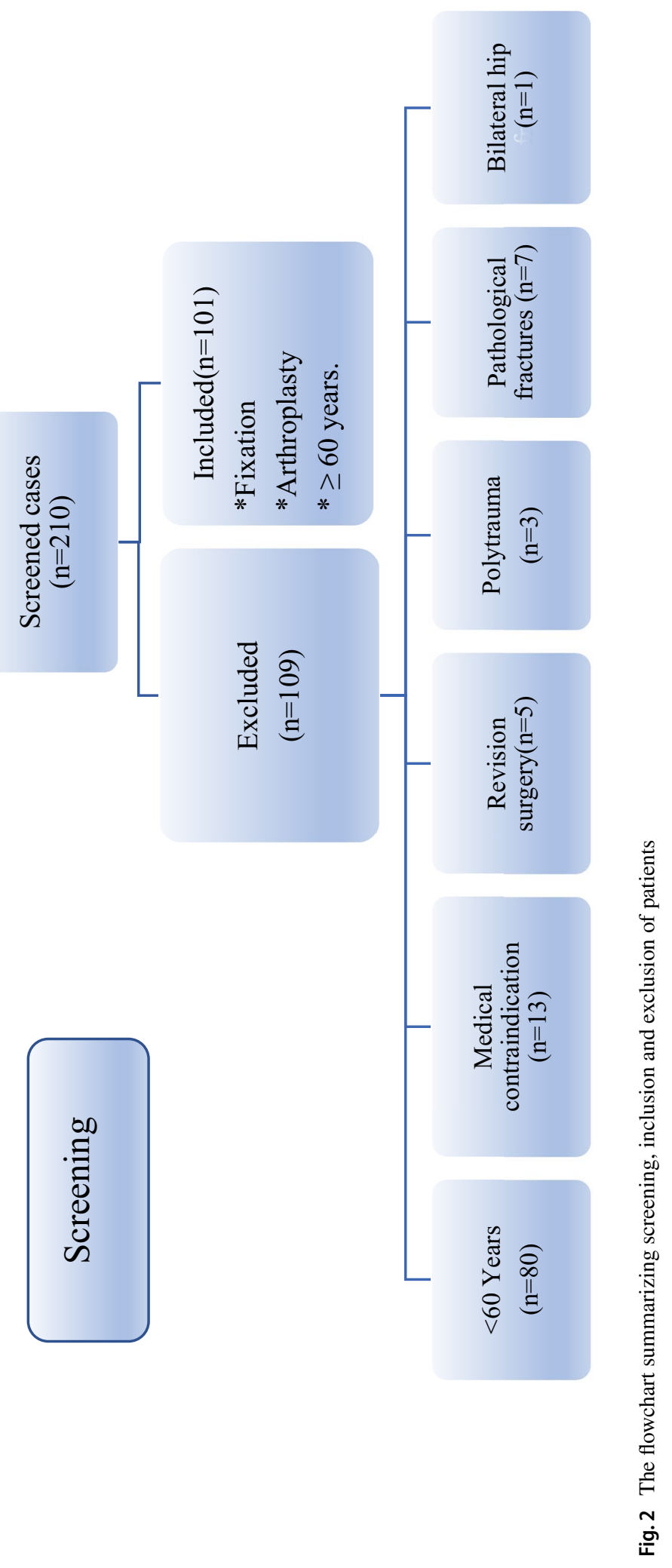


Table 2 Patients' variables and characteristics

\begin{tabular}{|c|c|c|}
\hline Patients' characteristics & Frequency & Percentage \\
\hline \multicolumn{3}{|l|}{ Surgical approach } \\
\hline Posterior & 7 & $6.9 \%$ \\
\hline Anterior/anterolateral & 17 & $17.1 \%$ \\
\hline Non-arthroplasty & 77 & $76 \%$ \\
\hline \multicolumn{3}{|l|}{ Pre-operative mobility/functional status } \\
\hline High functioning/ high demand & 14 & $13.8 \%$ \\
\hline $\begin{array}{l}\text { Moderate functioning (able to leave house } \\
\text { with or without assistance) }\end{array}$ & 35 & $34.6 \%$ \\
\hline $\begin{array}{l}\text { Low functioning (not able to leave house } \\
\text { with or without assistance) }\end{array}$ & 48 & $47.5 \%$ \\
\hline Non-ambulatory (bed dependent) & 4 & $\begin{array}{l}47.5 \% \\
3.9 \%\end{array}$ \\
\hline \multicolumn{3}{|l|}{ Cognitive impairment } \\
\hline Intact & 92 & $91.1 \%$ \\
\hline Mild cognitive dysfunction & 4 & $4 \%$ \\
\hline Moderate or severe cognitive dysfunction & 5 & $4.9 \%$ \\
\hline \multicolumn{3}{|l|}{ Post-operative delirium } \\
\hline Yes & 3 & $3 \%$ \\
\hline No & 98 & $97 \%$ \\
\hline \multicolumn{3}{|l|}{ Gender } \\
\hline Male & 52 & $51.5 \%$ \\
\hline Female & 49 & $48.5 \%$ \\
\hline \multicolumn{3}{|l|}{ Age } \\
\hline Mean & 75.6 years & \\
\hline Range & 60-96 years & \\
\hline \multicolumn{3}{|l|}{ Length of hospital stay } \\
\hline Mean & 12 days & \\
\hline Range & 2-74 days & \\
\hline
\end{tabular}

\section{Discussion}

The most important finding of this study was that the appropriateness rate in our patients was significantly lower than the AUC recommendations for the appropriate and maybe appropriate treatments; however, the rarely appropriate treatment was not statistically different compared with the AUC recommendation.

Furthermore, this study demonstrated a remarkable variation in the appropriateness of post-operative rehabilitation procedures for elderly hip fractures at our center. It was appropriate and in agreement with AUC recommendations only in a few procedures and, to a lesser extent, in the other rehabilitation procedures.

It is concerning that some rehabilitation procedures were not provided in a high percentage of patients at our institution, like home care therapy, delirium prevention, delirium management. The reasons might be due to the lack of evidence-based post-operative rehabilitation protocols for hip fractures in elderly patients at our institute, underestimation of the importance of these procedures by some surgeons, and/or the lack of the orthogeriatric service at our center. Consequently, implementation of AUC for post-operative rehabilitation of hip fractures in elderly could allow surgeons to explore all available and evidence-based rehabilitation options. It is a known fact from the literature and AUC guidelines that the post-operative rehabilitation procedures should be provided for any elderly patients with hip fractures to improve the quality of patient care by guiding the treating physicians in selecting an appropriate rehabilitation procedure. Hence, the variation in the post-operative rehabilitation for elderly patients with hip fractures should be decreased, and the patient care should be improved $[2,3,15,16]$.

Table 3 Actual rehabilitation procedures appropriateness and agreement with the AUC recommendations

\begin{tabular}{|c|c|c|c|c|c|c|c|c|}
\hline Rehabilitation Types & Provided & Not provided & Removed & Appropriate & Maybe & Rarely & $\begin{array}{l}\text { Agree- } \\
\text { mentwith } \\
\text { AUC }\end{array}$ & $p$ value \\
\hline DVT prophylaxis/use of anticoagulant & 101 & - & - & $101(100 \%)$ & - & - & $100 \%$ & - \\
\hline Multimodal pain Management & 101 & - & - & $101(100 \%)$ & - & - & $100 \%$ & - \\
\hline Osteoporosis Assessment \& Management & 73 & 28 & - & $73(72.2 \%)$ & - & - & $72.2 \%$ & $<.01$ \\
\hline Outpatient Occupational and Physical therapy & 30 & 17 & 54 & $30(63.8 \%)$ & - & - & $63.8 \%$ & .01 \\
\hline Weight bearing restriction & 59 & 42 & - & - & $1(1 \%)$ & $58(57.4 \%)$ & $58.4 \%$ & $<.001$ \\
\hline Range of motion restriction & 21 & 80 & - & - & $18(17.7 \%)$ & $3(3 \%)$ & $20.7 \%$ & $<.001$ \\
\hline Home care therapy & 13 & 39 & 49 & $13(25 \%)$ & - & - & $25 \%$ & $<.001$ \\
\hline $\mathrm{IRF}$ or $\mathrm{SNF} \dagger$ & 27 & - & 74 & $27(100 \%)$ & - & - & $100 \%$ & - \\
\hline Delirium prevention & 10 & 88 & 3 & $10(10.2 \%)$ & - & - & $10.2 \%$ & $<.001$ \\
\hline Delirium Management & 1 & 2 & 98 & $1(33.3 \%)$ & - & - & $33.3 \%$ & .25 \\
\hline
\end{tabular}

${ }^{\dagger}=$ Inpatient Rehabilitation or Skilled Nursing Facility 


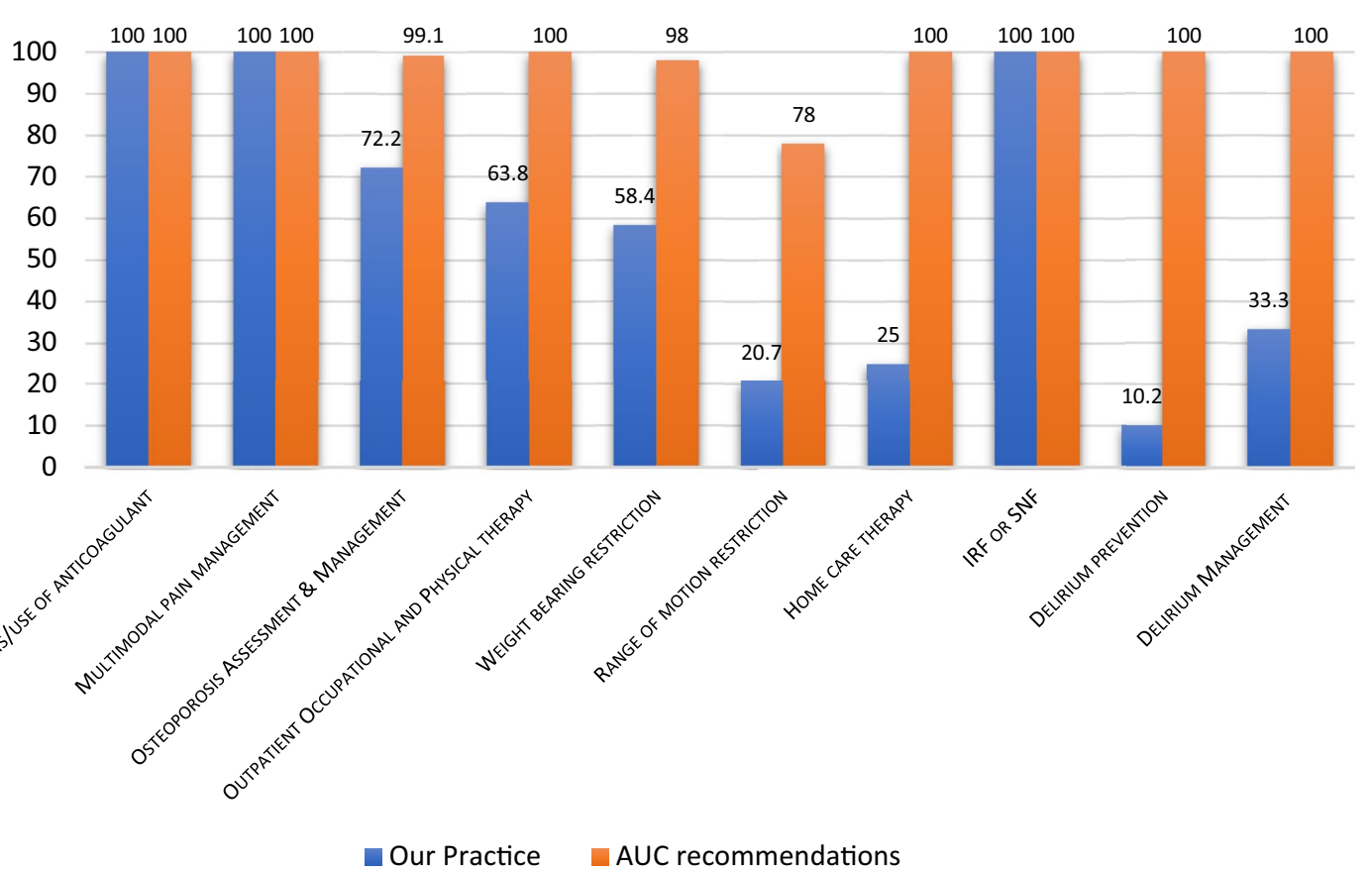

Fig. 3 Appropriateness of our rehabilitation procedures and AUC recommendations

Several authors have investigated the importance of the evidence-based post-operative rehabilitation protocols, and the orthogeriatric settings for the management of the elderly hip fracture [18-22].

Mark et al. demonstrated that hip fractures should be treated according to the most up-to-date evidence to achieve the best possible outcomes and optimal use of limited resources [18].

Beaupre et al. reported that the implementation of an evidence-based clinical pathway reduced the post-operative morbidity and did not affect in-hospital mortality or overall costs of inpatient care after hip fractures [19].

Adunsky et al. reported that the functional outcome of elderly hip fracture patients is better for those treated in the orthogeriatric setting, as compared with the common twosteps model of orthopedic surgery followed by transfer to a geriatric rehabilitation facility [20].

Siddiqi et al. emphasized that a proactive geriatric consultation may reduce delirium incidence and severity in patients undergoing surgery for hip fracture [21].

We also found that the application of patients' data into the free web-based AUC application made post-operative rehabilitation options for each case is relatively easy and simple to use. Most of the AUC rehabilitation therapies were provided at our center; however, not all the AUC scenarios were observed in our patients.

The availability of the AUC as a free web-based application is a valuable tool that can help the orthopedic surgeons to build their practice on a solid evidencebased data to improve the quality of care in such group of patients.

The AUC for the surgical treatment of knee osteoarthritis (OA) was recently evaluated, and concluded that AUC for the surgical treatment of knee OA can be applied easily in a clinical setting with $99 \%$ agreement was observed between the actual treatment of 100 patients and the AUC recommendations of appropriate treatment [22].

This study also has shown that the hip fractures were more common in male patients with a low prevalence of cognitive impairment and post-operative delirium.

The previous studies reported that the elderly hip fractures most commonly occurred in women with a higher prevalence of cognitive impairment and post-operative delirium [23-27].

These variations might be attributed to the health, cultural, and demographic variation across the countries and/ or the global geographic variations in hip fractures and cognitive impairment [28, 29].

In Qatar, the population characteristics are male predominance with a ratio of $2.6: 1$ because it is a newly growing country and depends mainly on male laborers from other countries and constitutes about $72 \%$ of the total population [30]. Additionally, patients with cognitive problems and other disabling condition tend to go back to their home country as they will be unable to work. Furthermore, the cognitive impairment and post-operative 
delirium might be underestimated in the pre-operative and post-operative assessment of our patients.

There were some drawbacks of AAOS published AUC for PORHFE: The appropriateness was not clearly defined for each recommendation of AUC, such as what is the alternative to the appropriateness ratings if the recommended procedure was not provided. For example, the range of motion restriction and weight-bearing restriction was always graded as maybe or rarely appropriate in all AUC scenarios; however, they did not specify how it graded the appropriateness if there were no restrictions.

Sherrington et al. reported in a randomized clinical trial that weight-bearing and non-weight-bearing exercise programs produce similar effects on strength, balance, gait, and functional performance among inpatients after hip fracture [31].

Home care therapy is a treatment that still controversial, where not all patients or hospitals could afford such treatment after the AUC amendment.

\section{Limitations}

This study has several limitations: the retrospective design of study, lack of comparative group, and patient outcome. The lack of sample size calculation, low level of evidence, and the cultural difference across the countries might affect the appropriateness ratings are other limitations of this study.

\section{Conclusions}

This study demonstrated that there is a remarkable variation in the appropriateness of the various post-operative rehabilitation procedures for elderly hip fracture. Additionally, the AUC application was easy to use and simple for identifying post-operative rehabilitation protocols for elderly hip fractures in the trauma setting, hence, we recommend to use it in the clinical practice.

Acknowledgements We would like to thank the Medical Research Center for their support and Dr. Abdullah Abunimer for his contribution. Approval for the study was obtained from the Institutional Research center and informed consent was exempted.

Author's contribution All authors contributed to the study conception and design. Material preparation, data collection and analysis were performed by Mohammed Adam, Eslam Alkaramany, Abdullah Murshid, Jawad Derbas and Abduljabbar Alhamoud. The first draft of the manuscript was written by Ghalib Ahmed Alhaneedi and all authors commented on previous versions of the manuscript. All authors read and approved the final manuscript.

Funding Open access funding provided by the Qatar National Library.
Data availability The datasets generated during and/or analyzed during the current study are available from the corresponding author on reasonable request.

\section{Declarations}

Conflicts of interest The authors declare that they have no conflicts of interest.

Ethics approval This article does not contain any studies with human participants or animals performed by any of the authors. Approval for the study was obtained from the Institutional Research center.

Open Access This article is licensed under a Creative Commons Attribution 4.0 International License, which permits use, sharing, adaptation, distribution and reproduction in any medium or format, as long as you give appropriate credit to the original author(s) and the source, provide a link to the Creative Commons licence, and indicate if changes were made. The images or other third party material in this article are included in the article's Creative Commons licence, unless indicated otherwise in a credit line to the material. If material is not included in the article's Creative Commons licence and your intended use is not permitted by statutory regulation or exceeds the permitted use, you will need to obtain permission directly from the copyright holder. To view a copy of this licence, visit http://creativecommons.org/licenses/by/4.0/.

\section{References}

1. Melton LJ (1996) Epidemiology of hip fractures: Implications of the exponential increase with age. Bone 18(3):S121-S125. https:// doi.org/10.1016/8756-3282(95)00492-0

2. Management of hip fractures in the elderly evidence-based clinical practice guideline. (2014) Available at: https://aaos.org/globa lassets/quality-and-practice-resources/hip-fractures-in-the-elder ly/management_of_hip_fractures_in_the_elderly-7-24-19.pdf. Accessed March 1, 2020

3. Roberts KC, Brox WT (2015) AAOS clinical practice guideline: management of hip fractures in the elderly. J Am Acad Orthop Surg 23(2):138-140. https://doi.org/10.5435/JAAOS-D-14-00433

4. Magaziner J, Hawkes W, Hebel JR et al (2000) Recovery from hip fracture in eight areas of function. J Gerontol A Biol Sci Med Sci 55(9):M498-M507. https://doi.org/10.1093/gerona/55.9.M498

5. Moyet J, Deschasse G, Marquant B, Mertl P, Bloch F (2019) Which is the optimal orthogeriatric care model to prevent mortality of elderly subjects post-hip fractures? A systematic review and meta-analysis based on current clinical practice. Int Orthop 43(6):1449-1454. https://doi.org/10.1007/s00264-018-3928-5

6. Marottoli RA, Berkman LF, Cooney LM (1992) Decline in physical function following hip fracture. J Am Geriatr Soc 40(9):861866. https://doi.org/10.1111/j.1532-5415.1992.tb01980.x

7. Jamal Sepah Y, Umer M, Khan A, UllahKhanNiazi A (2010) Functional outcome, mortality and in-hospital complications of operative treatment in elderly patients with hip fractures in the developing world. Int Orthop 34(3):431-435. https://doi.org/10. 1007/s00264-009-0803-4

8. Tidermark J, Zethraeus N, Svensson O, Törnkvist H, Ponzer $S$ (2002) Femoral neck fractures in the elderly: functional outcome and quality of life according to EuroQol. Qual Life Res 11(5):473-481. https://doi.org/10.1023/a:1015632114068

9. Jongjit J, Komsopapong L, Songjakkaew P, Kongsakon R (2003) Health-related quality of life after hip fracture in the elderly 
community-dwelling. Southeast Asian J Trop Med Public Health 34(3):670-674

10. Lyons AR (1997) Clinical outcomes and treatment of hip fractures. Am J Med 103(2):S51-S64. https://doi.org/10.1016/S00029343(97)90027-9

11. Civinini R, Paoli T, Cianferotti L et al (2019) Functional outcomes and mortality in geriatric and fragility hip fractures-results of an integrated, multidisciplinary model experienced by the "Florence hip fracture unit.” Int Orthop (SICOT) 43:187-192. https://doi. org/10.1007/s00264-018-4132-3

12. Barnes B, Dunovan K (1987) Functional Outcomes After Hip Fracture. Phys Ther 67(11):1675-1679. https://doi.org/10.1093/ $\mathrm{ptj} / 67.11 .1675$

13. Guccione AA, Fagerson TL, Anderson JJ (1996) Regaining functional independence in the acute care setting following hip fracture. Phys Ther 76(8):818-826. https://doi.org/10.1093/ptj/76.8. 818

14. Kumar VN, Redford JB (1984) Rehabilitation of hip fractures in the elderly. Am Fam Physician 29(1):173-180

15. Appropriate Use Criteri apost-operative time rehabilitation of hip fractures in the elderly. (2015) Available at: http://www.ortho guidelines.org/go/auc/default.cfm?auc_id=224956\&actionxm= Terms. Accessed April 8, 2020

16. Mounasamy V, Guy P, Kates SL (2017) Appropriate use criterpost-operative rehabilitation of low energy hip fractures in the elderly. J Am Acad Orthop Surg 25(1):e15-e17. https://doi.org/ 10.5435/JAAOS-D-16-00473

17. Fitch K, Bernstein SJ, Aguilar MD et al (2001) The RAND/UCLA appropriateness method user's manual. RAND Corporation, Santa Monica, CA

18. Mak JC, Cameron ID, March LM (2010) Evidence-based guidelines for the management of hip fractures in older persons: an update. Med J Aust 192(1):37-41. https://doi.org/10.5694/j.13265377.2010.tb03400.x[27-30]

19. Beaupre LA, Cinats JG, Senthilselvan A et al (2005) Reduced morbidity for elderly patients with a hip fracture after implementation of a perioperative evidence-based clinical pathway. Qual Saf Health Care 15(5):375-379. https://doi.org/10.1136/qshc. 2005.017095

20. Adunsky A, Lusky A, Arad M, Heruti RJ (2003) A comparative study of rehabilitation outcomes of elderly hip fracture patients: the advantage of a comprehensive orthogeriatric approach. J Gerontol A Biol Sci Med Sci 58(6):542-547. https://doi.org/10.1093/ gerona/58.6.m542

21. Siddiqi N, Stockdale R, Britton AM, Holmes J. (2007) Interventions for preventing delirium in hospitalized patients. Cochrane
Database of Systematic Reviews. Issue 2. Art. No. CD005563. https://doi.org/10.1002/14651858.CD005563.pub2.

22. Ahmed GO, ELSweify K, Ahmed AF (2020) Usability of the AAOS Appropriate Use Criteria (AUC) for the surgical management of knee osteoarthritis in clinical practice. Knee Surg Sports Traumatol Arthrosc Off J ESSKA 28(7):2077-2081. https://doi. org/10.1007/s00167-020-05908-7

23. Omsland K, Holvik K, Meyer E et al (2012) Hip fractures in Norway 1999-2008: time trends in total incidence and second hip fracture rates: a NOREPOS study. Eur J Epidemiol 27(10):807814. https://doi.org/10.1007/s10654-012-9711-9

24. Lieberman D1, Lieberman D (2004) Rehabilitation following hip fracture surgery: a comparative study of females and males. Disabil Rehabil 26(2):85-90. https://doi.org/10.1080/1965382803 10001629660

25. Johnell O, Kanis JA (2004) An estimate of the worldwide prevalence, mortality, and disability associated with hip fracture. Osteoporos Int 15(11):897-902. https://doi.org/10.1007/ s00198-004-1627-0

26. Seitz DP, Adunuri N, Gill SS, Rochon PA (2011) Prevalence of dementia and cognitive impairment among older adults with hip fractures. J Am Med Dir Assoc 2(8):556-546. https://doi.org/10. 1016/j.jamda.2010.12.001

27. Guo Y, Jia P, Zhang J, Wang X, Jiang H, Jiang W (2016) Prevalence, and risk factors of post-operative time delirium in elderly hip fracture patients. J Int Med Res 44(2):317-327. https://doi. org/10.1177/0300060515624936

28. Dhanwal DK, Dennison EM, Harvey NC, Cooper C (2011) Epidemiology of hip fracture: worldwide geographic variation. Indian J Orthop 45(1):15-22. https://doi.org/10.4103/0019-5413.73656

29. Michael Lewiecki E, Wright NC, Curtis JR et al (2018) Hip fracture trends in the United States, 2002 to 2015. Osteoporos Int 29:717-722. https://doi.org/10.1007/s00198-017-4345-0

30. Psa.gov.qa. 2020. Monthly figures on total population. [online] Available at: Psa.gov.qa. 2020. Monthly Figures On Total Population. [online] Available at: <https://www.psa.gov.qa/en/stati stics1/StatisticsSite/Pages/Population.aspx $>$ [Accessed 23 October 2020]

31. Sherrington C, Lord SR, Herbert RD (2003) A randomized trial of weight-bearing versus non-weight-bearing exercise for improving physical ability in inpatients after hip fracture. Aust J Physiother 49(1):15-22. https://doi.org/10.1016/s0004-9514(14)60184-7

Publisher's Note Springer Nature remains neutral with regard to jurisdictional claims in published maps and institutional affiliations. 